Meta

Journal des tradlucteurs

Translators' Journal

\title{
Il n'y a pas de suffixe -ateur en français, voyons'! (II)
}

\section{Antoine Di-Lillo}

Volume 27, numéro 4, décembre 1982

URI : https://id.erudit.org/iderudit/002568ar

DOI : https://doi.org/10.7202/002568ar

Aller au sommaire du numéro

Éditeur(s)

Les Presses de l'Université de Montréal

ISSN

0026-0452 (imprimé)

1492-1421 (numérique)

Découvrir la revue

Citer cet article

Di-Lillo, A. (1982). Il n'y a pas de suffixe -ateur en français, voyons'! (II). Meta, 27(4), 404-420. https://doi.org/10.7202/002568ar d'utilisation que vous pouvez consulter en ligne.

https://apropos.erudit.org/fr/usagers/politique-dutilisation/ 


\section{ÉTUDES \\ TERMINOLOGIQUES \\ ET LINGUISTIQUES}

\section{IL N'Y A PAS DE SUFFIXE -ATEUR EN FRANÇAIS, VOYONS ! (II)}

\section{PRÉAMBULE}

Nous faisions état, dans la partie précédente, des études faites sur le sujet que nous traitons et du consensus qui règne sur le statut à accorder aux formes agentives en -eur: Les auteurs de dictionnaires, de grammaires, ou d'études sur la morphologie du français s'accordent à dire que toutes les formes en -eur sont déverbales et que les formes en -t-eur (X-t-eur), sous-ensemble des formes en -eur, sont issues du radical verbal par adjonction d'un suffixe, généralement -ateur mais quelquefois -iteur ou -teur, prétendue variante morphologique du suffixe -eur. Or, nous maintenons que seules les formes finissant directement en -eur (X-eur) peuvent dériver d'un verbe (ex. : assureur) bien que quelques-unes puissent dériver de bases nominales monomorphémiques (ex. : chroniqu-eur).

C'est dans la première partie de cet article, donc, que nous avons analysé les X-eur et montré de quel type de base elles pouvaient dériver, bases verbales pour la plupart, bases nominales pour certaines. Il nous fallait commencer par étudier ces formes, assez transparentes morphologiquement, pour ensuite nous attaquer aux X-t-eur, plus complexes, et montrer que l'analyse retenue pour les $\mathrm{X}$-eur ne peut convenir pour les X-t-eur.

Dans la seconde partie qui suit, nous nous proposons d'examiner de près les $\mathrm{X}$-t-eur. Nous constaterons que vouloir dériver ces formes de bases verbales nous mène à une complexité extrême car ces formes ne dérivent pas de radicaux verbaux mais de formes nominales suffixées en -ion. Nous invoquerons essentiellement quatre arguments en faveur de notre hypothèse : 1) Les X-teur ont pratiquement toujours un corrélat en -t-ion (X-t-ion). 2) Il y a une correspondance morphologique absolue entre X-t-eur et X-t-ion. 3) Notre hypothèse de la base X-t-ion est la seule qui puisse rendre compte de l'existence de paires du genre receveur récepteur. 4) Notre hypothèse est la seule qui permette d'expliquer la dérivation des X-t-eur sans corrélats verbaux.

Notre analyse est très simple puisqu'elle n'a recours à aucune règle d'allomorphie ni du radical ni du suffixe, elle fait uniquement appel à une règle de troncation de -ion et à une règle de réajustement, qui est d'ailleurs indépendamment motivée pour le français, convertissant /s/ en /t/ devant -eur. 


\section{NOMS D'AGENT X-T-EUR}

\subsection{Problèmes de description des $X$-t-eur}

\subsubsection{La prétendue variante -ateur et autres allomorphes}

En examinant les dérivés de cette classe, on a tôt fait de s'apercevoir que le plus grand nombre se termine en -ateur. Voici quelques représentants de cette classe très nombreuse. Comme précédemment, nous faisons suivre chaque formation du corrélat verbal et nominal. Il est à noter qu'à chaque $X$ - $t$-eur correspond une X-t-ion.

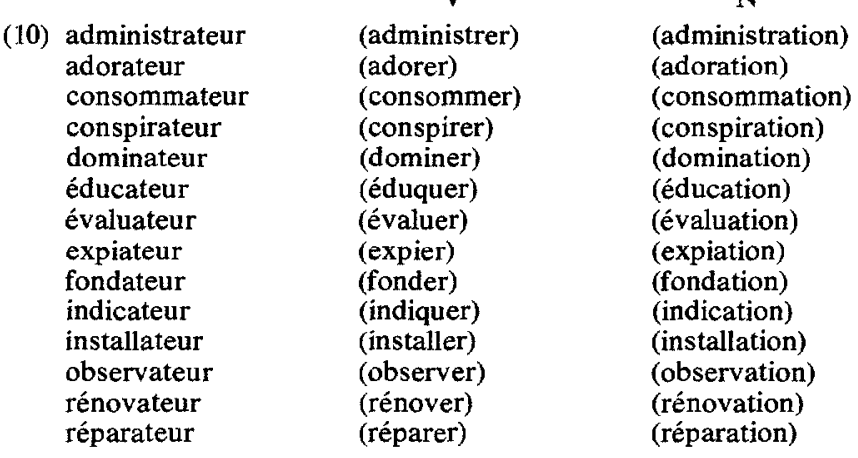

Si nous analysons les formes en (10) comme le font Dubois (1962) ou Bourbeau (1976), nous dirons qu'elles sont issues des verbes correspondants par suffixation de -ateur, variante morphologique du suffixe-eur. Nous aurions de cette manière administr-ateur, ador-ateur, consomm-ateur. Avant de voir ce que cette analyse implique, examinons les formes suivantes:

\begin{tabular}{|c|c|c|c|c|}
\hline & & & V & $\mathbf{N}$ \\
\hline \multirow[t]{12}{*}{ (11) } & (a) & compositeur & (composer) & (composition) \\
\hline & & inhibiteur & (inhiber) & (inhibition) \\
\hline & & répétiteur & (répéter) & (répétition) \\
\hline & (b) & distributeur & (distribuer) & (distribution) \\
\hline & & expéditeur & (expédier) & (expédition) \\
\hline & (c) & classificateur & (classifier) & (classification) \\
\hline & & excommunicateur & (excommunier) & (excommunication) \\
\hline & & multiplicateur & (multiplier) & (multiplication) \\
\hline & & sanctificateur & (sanctifier) & (sanctification) \\
\hline & (d) & liquéfacteur & (liquéfier) & (liquéfaction) \\
\hline & & putréfacteur & (putréfier) & (putréfaction) \\
\hline & & torréfacteur & (torréfier) & (torréfaction) \\
\hline
\end{tabular}

Étant donné que les formes en (11) finissent en -teur, même si ce n'est pas en -ateur, et que l'élément en -teur semble conférer à la forme dans son ensemble la même valeur sémantique que -ateur, il peut être raisonnable d'analyser, comme Dubois ou Bourbeau, cet élément suffixal comme une variante de -ateur. Cette variante serait donc-iteur pour les formes (11) (a), -teur pour (11) (b), -cateur pour (11) (c) et -acteur (ou -cteur ?) pour (11) (d). (Les trois formes de (d) semblent poser un problème : le radical du verbe est respectivement liquéfi-, putréfi- et torréfi-; pourtant la voyelle finale /i/ n'apparaît pas dans le dérivé. Doit-on postuler l'effacement de /i/ devant le suffixe-acteur? 
Ou doit-on admettre que /i/ devient /a/ dans le contexte le moment nous tiendrons compte des deux possibilités sans trancher).

Nous venons de supposer que -ateur, qu'on retrouve le plus fréquemment dans les formes en -teur, a quatre allomorphes qui sont -iteur, -teur, -cateur et acteur, (ou -cteur). Or, si nous admettons que ces formes suffixales sont des variantes de -ateur, il faut, par voie de conséquence, admettre également qu'elles sont des variantes morphologiques du suffixe -eur.

À présent que nous avons dénombré six variantes d'un même suffixe, en l'occurrence -eur, nous allons voir si la distribution des allomorphes du suffixe -eur est explicable et prévisible; car il faudrait pouvoir expliquer l'alternance entre -eur d'une part et les variantes en -teur d'autre part; il faudrait même pouvoir rendre compte de la distribution des variantes en -teur.

Une première tentative consisterait à opérer une distinction par groupes de conjugaison des verbes auxquels s'adjoint le suffixe. Nous constatons que -ateur s'adjoint exclusivement à des verbes du 1er groupe (en -er) : administrer/administrateur, adorer/adorateur, consommer/consommateur. Malheureusement, nous devons dire la même chose de -iteur (composer/compositeur, inhiber/inhibiteur, répéter/répétiteur), de -teur (distribuer/distributeur, expédier/expéditeur), de -cateur (sanctifier/sanctificateur, multiplier/multiplicateur) et de -acteur (ou -cteur) (liquéfier/liquéfacteur, putréfier/putréfacteur).

Comme nous venons de le voir, cette approche ne permet pas d'expliquer ce qui déclenche l'allomorphie :

$$
\begin{array}{ll} 
& \text {-iteur } \\
\text {-ateur } \rightarrow \quad \text {-teur } \\
\text {-cateur } \\
\text {-acteur ou -cteur }
\end{array}
$$

mais encore elle ne peut justifier la présence de -eur, d'un côté, et des variantes en -teur, de l'autre, puisque -eur sélectionne également comme bases des radicaux de verbes du ler groupe (assurer/assureur, balayer/balayeur). Toutefois, ce qui n'est pas le cas pour les variantes en -teur, -eur peut s'adjoindre à des radicaux de n'importe quel groupe de conjugaison (avertir/avertisseur, boirelbuveur, battrelbatteur, etc.).

Cette tentative s'avérant infructueuse, une autre approche serait de voir si la forme de la variante n'est pas tributaire de la finale du radical, voyelle ou consonne. Il serait, a priori, raisonnable de penser que -eur ou -ateur, commençant par une voyelle, pourraient sélectionner uniquement des radicaux finissant par une consonne, les variantes commençant par une consonne s'adjoindraient aux radicaux finissant par une voyelle, et que peut-être même la forme de chaque variante dépendrait de la voyelle ou de la consonne du radical. Malheureusement nous ne pouvons, non plus, retenir cette solution; le suffixe -ateur, il est vrai, est associé aux radicaux verbaux finissant par une consonne : adorateur, fond-ateur, incub-ateur etc., mais il est aussi associé aux radicaux verbaux finissant par une voyelle : continu-ateur, cré-ateur, énuclé-ateur, conciliateur, spoli-ateur. La seule variante en -teur qui s'adjoint exclusivement à une base finissant par une consonne est -iteur : compos-iteur, répét-iteur. Les trois autres variantes en -teur: -teur, -cateur et -acteur (ou -cteur) sélectionnent 
des bases finissant par une voyelle: distribu-teur, purif-cateur, liquéf(i)-acteur. Comble de confusion, les verbes en -fier sont associés aussi bien à la variante cateur qu'à la variante -acteur: purifier/purificateur, liquéfier/liquéfacteur, le seul critère semblant jouer est le type de voyelle qui précède le /f/ du radical (Xifier/Xéfier) : si c'est/i/, la variante sera -cateur, si c'est /e/, ce sera-acteur. Quant à -eur, on s'aperçoit qu'elle s'accommode de tous les contextes possibles et qu'elle peut concurrencer-ateur, ou les autres variantes en -teur, comme en témoignent les exemples suivants, toutes des formes attestées qui coexistent:

$\begin{array}{llll}\text { (12) } \begin{array}{l}\text { adapt-ateur } \\ \text { anglicis-ateur }\end{array} & \sim \text { adapt-eur } & \text { modifi-cateur } & \sim \text { modifi-eur } \\ \text { capt-ateur } & \sim \text { anglicis-eur } & \text { programm-ateur } & \sim \text { program-eur } \\ \text { compens-ateur } & \sim \text { compens-eur } & \text { quantifi-cateur } & \sim \text { quantifi-eur } \\ \text { condens-ateur } & \sim \text { condens-eur } & \text { transform-ateur } & \sim \text { transform-eur }\end{array}$

Le tableau suivant résumera les contextes d'apparition de -eur et de ses prétendues variantes :

(13)

\begin{tabular}{|c|c|c|c|c|c|}
\hline & \multicolumn{3}{|c|}{$\begin{array}{l}\text { DISTRIBUTION PAR GROUPES } \\
\text { DE CONJUGAISON }\end{array}$} & \multicolumn{2}{|c|}{$\begin{array}{l}\text { DISTRIBUTION PAR } \\
\text { TERMINAISONS } \\
\text { DU RADICAL }\end{array}$} \\
\hline & $1^{\mathrm{er}}$ & $2 e$ & $3 e$ & voy. & cons. \\
\hline$-e u r$ & $\mathrm{X}$ & $X$ & $X$ & $\mathrm{X}$ & $\mathbf{X}$ \\
\hline -ateur & $\mathrm{X}$ & & & $\mathrm{X}$ & $\mathrm{X}$ \\
\hline -iteur & $\mathrm{X}$ & & & & $\mathrm{X}$ \\
\hline -teur & $\mathrm{X}$ & & & $\mathrm{X}$ & \\
\hline -cateur & $X$ & & & $X$ & \\
\hline $\begin{array}{l}\text {-acteur } \\
\text { (ou-cteur) }\end{array}$ & $X$ & & & $\mathrm{X}$ & \\
\hline
\end{tabular}

Finalement, un coup d'œil aux quelques exemples ci-dessous finira de nous convaincre de l'imprévisibilité de la forme du suffixe :
(14) adorer/adorateur _ mais: dorer/doreur (*dorateur)
compenser/compensateur - mais: penser/penseur (*pensateur)
composer/compositeur _ mais: poser/poseur (*positeur)
consommer/consommateur - mais: assommer/assommeur (*assommateur)
expédier/expéditeur _ - mais: répudier/répudiateur (*répuditeur, *expédiateur)
instituer/instituteur - mais : perpétuer/perpétuateur (*perpétuteur, *instituateur)
putréfier/putréfacteur - mais : sanctifier/sanctificateur ("sanctifacteur, *putréficateur)
réaliser/réalisateur _ mais: analyser/analyseur, (*analysateur)
répéter/répétiteur _ mais : rouspéter/rouspéteur (*rouspétiteur)

Ainsi, si l'on tient à suivre l'analyse traditionnelle et à considérer les $X$-t-eur comme des formes déverbales, il faut admettre qu'elles posent des problèmes de traitement pour n'importe quel modèle. Dans le modèle d'Aronoff, on serait contraint de renoncer à une règle de formation de mots, puisqu'on ne réussirait pas à établir convenablement les règles d'allomorphie du suffixe -eur (-ateur, -teur, -iteur, etc.). Il faut supposer que toutes les formes X-t-eur seraient listées au lexique et qu'elles seraient reliées à leur corrélat verbal par des règles de redondance, traitement dont nous verrons un peu plus loin la 
lourdeur. Si l'on adopte le cadre de Lieber, il faudrait, pour rendre compte de ces formes, inscrire dans l'entrée lexicale du verbe deux radicaux allomorphes: un radical simple qui servirait de base à la dérivation de type $\mathrm{X}$-eur et un radical dérivé (savant) X-t- pour les formes X-t-eur. On aurait ainsi recours à l'allomorphie du radical plutôt qu'à celle du suffixe. Pourtant le problème n'est pas si simple, même pour ce modèle. Si nous considérons par exemple que administrat- est le radical long dérivé de administr-, puisque nous avons administrateur, il faut aussi admettre que, par exemple, comparat-, cumulat-, dormit-, fugit-, vomit- sont les dérivés de compar-, cumul-, dorm-, fui-, vomi-, puisque nous avons comparatif, cumulatif, dormitif, fugitif, et vomitif; or nous n'avons pas *comparateur, *cumulateur, *dormiteur, etc. Cela obligerait Lieber à marquer tous les radicaux dérivés qui prennent -eur, ce qui rendrait le traitement extrêmement complexe. (Nous anticipons ici sur l'hypothèse que nous formulerons un peu plus loin, mais nous dirons qu'en fait la X-t-eur existe parce que la X-t-ion existe et que puisque nous n'avons pas ${ }^{*}$ fugition ou *vomition, nous ne pouvons avoir *fugiteur ou *vomiteur.)

\subsubsection{Les X-t-eur à radicaux latins}

Avant de nous attacher à trouver une solution définitive considérons les exemples suivants qui constituent également des noms d'agents X-t-eur.

(15)

adjudicateur
approbateur
constructeur
correcteur
directeur
instructeur
producteur
promoteur
récepteur
rédacteur
séducteur
souscripteur
traducteur

\begin{tabular}{ll}
$\quad \mathrm{V}$ & \multicolumn{1}{c}{$\mathrm{N}$} \\
(adjuger) & (adjudication) \\
(approuver) & (approbation) \\
(construire) & (construction) \\
(corriger) & (correction) \\
(diriger) & (direction) \\
(instruire) & (instruction) \\
(produire) & (production) \\
(promouvoir) & (promotion) \\
(recevoir) & (réception) \\
(rédiger) & (rédaction) \\
(séduire) & (séduction) \\
(souscrire) & (souscription) \\
(traduire) & (traduction)
\end{tabular}

Les formes ci-dessus se caractérisent par le fait qu'il y a une différence assez nette entre la morphologie du verbe et celle du mot en -teur correspondant. Nous sommes témoins, dans ces mots, de l'écart qui s'est creusé entre latin et français, car nous avons affaire à des radicaux latins. Si les formes que nous avons vues précédemment en (11) ne pouvaient dériver par une règle de formation de mot, dans le cadre d'Aronoff, celles-ci le peuvent encore moins puisqu'en plus de la règle d'allomorphie qu'il faut postuler pour le suffixe, il faut avoir recours à des règles d'allomorphie du radical.

Nous allons montrer ci-dessous ce que serait le traitement des formes en -teur que nous avons vues jusqu'ici dans le modèle d'Aronoff. Nous voulons toutefois signaler que le traitement que préconise Aronoff pour les formes en -or de l'anglais ne peut s'appliquer au français pour les formes en -teur parce qu'en anglais le suffixe productif des noms d'agent n'est pas -or mais -er (eat-er, walk-er, etc.); par contre, en français, nous n'avons qu'un seul et même suffixe: -eur. 
Étant donné qu'il n'existe, comme nous l'avons vu, aucun critère permettant de rendre compte de la distribution de -eur d'un côté et de ses variantes en -teur de l'autre, et qu'en plus il est impossible de prévoir, pour chaque cas, la variante en -teur spécifique à partir de la forme du radical, Aronoff serait contraint d'entrer tous les mots en -teur au lexique et les relier aux radicaux verbaux par des règles de redondance.

On peut supposer que les radicaux verbaux qui prennent les variantes suffixales en -teur seront marqués au lexique d'un trait qui pourrait être, par exemple, [+latin]. Mais comme ce trait à lui seul ne peut rendre compte de la distribution des variantes en -teur, il faudra lui ajouter un autre trait. Nous supposerons que les radicaux prenant -ateur auront le trait [+latin I], ceux prenant -teur [+latin II] et ceux prenant -iteur [+latin III]. On peut à présent proposer la règle d'allomorphie du suffixe -eur suivante :

$\begin{array}{llll}\text { (16) -eur } & \text {-ateur } & \text {-teur } & \text { latin I } \\ & \text {-iteur } & & \text { latin II } \\ & & \text { latin III }\end{array}$

Le schéma de règles (16) énonce que -ateur choisira le radical verbal marqué [+ latin I], -teur celui marqué [+ latin II] et ainsi de suite. Ces règles, qui n'ont aucun statut, ne peuvent intervenir productivement dans la dérivation des formes en -teur.

Il faut maintenant postuler pour les formes de (15) des règles d'allomorphie du radical; Aronoff noterait que même si l'allomorphie du radical ne peut $s^{\prime}$ 'expliquer par la simple forme de $X$, où $X$ est le radical simple, l'allomorphie est constante dans le paradigme de verbes partageant le même morphème latin :

(17) a) constructeur/construire

(Xtruct-/Xtrui)

destructeur/détruire

b) correcteur/corriger

(Xrect-/Xrig)

directeur/diriger

c) inducteur/induire producteur/produire

d) descripteur/décrire souscripteur/souscrire

(Xduct-/Xdui)

(Xcript-/Xcri)

Les règles d'allomorphie qui porteront sur ces morphèmes latins auront d'une façon simplifiée la forme suivante :

(18) a) trui $\rightarrow$ truct $/ \longrightarrow+$ eur

b) rig $\rightarrow$ rect , $\square$ +eur

c) dui $\rightarrow$ duct $/ \longrightarrow$ +eur

d) cri $\rightarrow$ cript,$\quad$ + eur

Comme on peut le constater, le traitement des formes en -teur à partir des radicaux verbaux nécessite, dans le modèle d'Aronoff, le déploiement d'un arsenal de règles si l'on veut rendre compte de toutes les variantes, règles qui n'ont qu'un statut de redondance.

\subsubsection{Les $\mathrm{X}$-t-eur où $\mathrm{X}$ n'existe pas}

Nous voici parvenu maintenant au dernier groupe de mots faisant partie de la classe $\mathrm{X}$-t-eur. En voici les exemples : 
(19) auditeur compétiteur

déprédateur

duplicateur

exacteur

excavateur

générateur

inquisiteur

investigateur

locomoteur

locuteur

médiateur

migrateur

perquisiteur

prestidigitateur

rédempteur

régulateur

ségrégateur

\begin{tabular}{cl}
$\mathrm{V}$ & \multicolumn{1}{c}{$\mathrm{N}$} \\
& (audition) \\
(compétition) & (déprédation) \\
& (duplication) \\
& (exaction) \\
& (excavation) \\
& (génération) \\
& (inquisition) \\
& (investigation) \\
& (locomotion) \\
& (locution) \\
& (médiation) \\
& (migration) \\
& (perquisition) \\
& (prestidigitation) \\
(peremption) & (régulation) \\
& (ségrégation)
\end{tabular}

Comme nous pouvons le constater, ces formes ne peuvent être associées à aucun verbe sauf pour perquisiteur dont perquisitionner dérive de la forme en -tion. Pourtant, elles peuvent être perçues comme agentives et même si le découpage suffixe/base pose a priori quelque difficulté, l'élément en -teur confère à la forme la valeur sémantique qui en fait un nom d'agent.

Le fait que ces formes ne peuvent être associées à aucun verbe constitue pour une analyse ayant comme point de départ le verbe un problème insurmontable. Le modèle d'Aronoff prédirait, à juste titre, que la dérivation des formes X-t-eur à partir de bases verbales, n'est pas productive; le modèle d'Aronoff n'est pas en cause mais c'est que, vues sous cet angle, les données sont incroyablement compliquées à décrire. Quant à Lieber (1980), si nous passons sur le problème de la représentation sémantique à donner à ces racines du genre audit- ou compétit-, qui ne peuvent être considérées comme radicaux dérivés, et sur celui de la catégorie à leur assigner ${ }^{1}$ (nous supposerons qu'elle les considérerait comme racines verbales), le modèle ne peut rendre compte de ces formes que par des règles morpho-lexicales, genre de règles de redondance, comme il le ferait d'ailleurs pour toutes les $\mathrm{X}$-t-eur que nous avons vues jusqu'à présent.

On peut donc constater que l'inexistence, dans de nombreux cas, des bases verbales porte un coup sérieux à toute analyse qui postule que les formes X-t-eur sont dérivées de verbes et cela vaut pour n'importe quel modèle morphologique.

\subsection{Hypothèse de la dérivation des $X$-t-eur à partir des $X$-t-ion}

Après avoir examiné tous les groupes de mots qui constituent cette grande classe de mots $\mathrm{X}$-t-eur, nous pouvons tirer les conclusions suivantes: les X-t-eur se distinguent des X-eur par le fait qu'elles sont plus complexes et qu'il est impossible de prédire à partir de la simple forme de $\mathrm{X}$, où $\mathrm{X}=\mathrm{V}$, la forme du suffixe et la présence ou l'absence d'augments. Nous avons vu que les $\mathrm{X}$-eur sont par contre parfaitement régulières. Si pour les X-eur X est un verbe existant, ce n'est pas toujours le cas pour les $\mathrm{X}$-t-eur où $\mathrm{X}$ peut ne pas

1. Chez Lieber, les entrées lexicales sont organisées en catégories lexicales majeures. 
exister. Les formes $\mathrm{X}$-t-eur posent donc de graves problèmes de description quel que soit le modèle qu'on adopte.

L'analyse traditionnelle, qui considère les X-t-eur comme déverbales formées par adjonction d'une variante du suffixe -eur, est incapable donc d'expliquer simplement, sur un plan synchronique, la distribution des allomorphes de ce suffixe; ce qui est plus grave, elle ne peut expliquer l'existence des X-t-eur n'ayant pas de corrélats verbaux (auditeur, compétiteur, déprédateur, etc.).

Nous allons tenter de démontrer que pour rendre compte synchroniquement de ces formes, d'une manière simple et efficace, il faut avoir recours à une analyse différente de celle qui a été présentée pour les X-eur. Nous montrerons que les X-t-eur, en français contemporain, sont dérivées de leur corrélat nominal $\mathrm{X}$-t-ion et non de verbes comme le veulent généralement les auteurs qui se sont intéressés à ces formes, même ceux qui ont adopté un cadre générativiste (Bourbeau 1976, Zwanenburg 1980). Le seul à notre connaissance qui ait mentionné le fait que les X-t-eur dérivent de formes en -tion est Goosse (1975) qui, dans une plaquette d'une soixantaine de pages consacrée aux néologismes, faisait la remarque suivante :

Parmi les 540 mots que j'ai considérés comme dérivés d'une base française, parce qu'il n'y a pas d'opposition de radical, un certain nombre sont en réalité à ranger ici : «contestateur» ne vient pas de «contester» mais de «contestation», par substitution de suffixe. Ceci confirme les observations déjà faites que la dérivation française s'opère surtout à partir de mots déjà suffixés. (p. 41)

Goosse nous dit aussi, à la même page, parlant du radical latin dans des formes du genre concepteur:

Ce radical savant peut ne pas être représenté en français, mais le plus souvent, on le trouve déjà dans d'autres dérivations. Et, dans la réalité, le nouveau dérivé n'est pas obtenu par un recours au latin, mais il est tiré des dérivés déjà introduits en français. C'est-à-dire qu'il s'agit d'une substitution de suffixes.

Selon nos propres observations, la dérivation française ne s'opère pas «surtout à partir de mots déjà suffixés », mais surtout à partir de mots déjà affixés, étant donné le nombre, également important, de mots déjà préfixés qui servent de base à la formation d'autres mots en français, comme nous le verrons plus loin.

Nous montrerons, après avoir donné nos arguments en faveur d'un tel traitement, quels sont les mécanismes que cette analyse met en jeu dans un modèle à la Aronoff (1976) ou à la Lieber (1980).

Nos premiers arguments se fondent sur le parallélisme étroit et constant entre forme en -teur et forme en -tion: en effet, premièrement, un rapide examen des tableaux que nous avons présentés révèle qu'il existe pour chaque mot en -teur un corrélat en -tion. Deuxièmement, peu importe l'écart qui s'est creusé entre le radical du mot en -tion et celui du corrélat verbal, le radical du mot en -teur a exactement la même morphologie que celui du mot en -tion: administrateur/administration, répétiteur/répétition, distributeur/distribution, classificateur/classification, liquéfacteur/liquéfaction, adjudicateur/adjudication, auditeur/audition. La seule exception que nous connaissons, qui nous a été mentionnée par Y.-C. Morin, est celle de coordonnateur/coordination. 
Nous avons, cependant, coordinateur (voir Petit Robert, p. 350) qui est, lui, le dérivé «légitime» du mot en -tion. À notre avis coordonnateur a été dérivé sur le modèle suggéré par le mot coordination, c'est-à-dire à partir d'un radical de type Xat, où X est le radical simple et Xat le radical dérivé, mais en donnant à $\mathrm{X}$ la morphologie du radical de coordonner. Ce serait en quelque sorte une forme «hybride». Ce cas peut être considéré comme l'exception qui confirme la règle de correspondance morphologique.

Le parallélisme que nous venons de souligner, et qui est manifeste si l'on considère les règles de redondance de Bourbeau qui s'appliquent sans exception à la fois aux dérivations en -teur et en -tion, nous fournit les premiers arguments en faveur de l'analyse que nous proposons. C'est en vertu de ce parallélisme que les locuteurs contemporains du français analysent les formes X-t-eur comme dérivées des X-t-ion.

Nous ouvrons ici une parenthèse pour répondre à une objection possible découlant de ce premier argument: pourquoi les locuteurs ne dériveraient-ils pas plutôt les X-t-ion à partir des X-t-eur correspondantes, au lieu de faire le contraire comme nous le suggérons? Cela pourrait expliquer également le parallélisme qui existe entre les formes respectives. Il nous faut toutefois rejeter cette hypothèse pour une importante raison de nature empirique: il y a relativement très peu de formes X-t-eur attestées sans corrélat X-t-ion (nous aurons l'occasion de présenter les exceptions dans la section suivante), alors qu'il y a une quantité considérable de X-t-ion sans corrélat X-t-eur. Cette observation se vérifie, semble-t-il par le raisonnement: le nom d'action peut exister sans qu'on attribue nécessairement cette action à un agent particulier; qu'on considère les exemples ci-dessous où aucun agent ne peut être associé : abomination, affabulation, altercation, circonvolution, congrégation, électrocution, exclamation, filiation, foliation, inflation, interaction, libation, médication, résignation, sensation, etc. Cependant, si le nom d'agent existe, il y a de fortes probabilités pour que le nom d'action existe également.

Comme autre argument en faveur de notre hypothèse, nous ferons remarquer l'existence des paires suivantes :

(21) adapteur

angliciseur

capteur

compenseur

condenseur

modifieur

programmeur

quantifieur

receveur

sélectionneur

spécifieur

transformeur

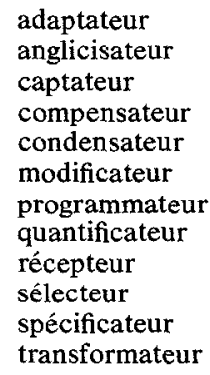

(adapter/adaptation)
(angliciser/anglicisation)
(capter/captation)
(compenser/compensation)
(condenser/condensation)
(modifier/modification)
(programmer/programmation)
(quantifier/quantification)
(recevoir/réception)
(sélectionner/sélection)
(spécifier/spécification)
(transformer/transformation) etc.

(transformer/transformation) etc.

Si nous avions affaire à une seule base verbale pour la dérivation des formes agentives en -eur, comment pourrait-on expliquer l'existence de ces paires? Si l'on suppose que X-t-ion est la base pour la forme X-t-eur, on s'explique facilement leur existence. Ce qui confirme encore cette hypothèse des deux bases, c'est qu'on ne trouve jamais des paires du genre compenseur $\sim$ compen- 
sateur si le X-t-ion n'existe pas (on n'aura jamais ainsi penseur $\sim$ *pensateur car on n'a pas *pensation).

Un dernier argument pour une dérivation des formes en -teur à partir des formes nominales en -tion est qu'il y a des X-t-eur qu'on ne peut associer à aucun verbe mais qui ont, pourtant, un corrélat en -tion comme en témoignent les exemples de (19). Si l'on veut rendre compte de ces dérivations, on doit nécessairement opérer à partir de la forme en -tion. Notre hypothèse, qui a le mérite d'être la plus simple pour la dérivation des X-t-eur, a de plus l'avantage de capter la productivité de la dérivation des $\mathrm{X}$-t-eur à partir des X-t-ion, alors que les autres analyses en se fondant sur le verbe ne peuvent que rendre compte de ces formes par des règles qui doivent forcément avoir un caractère de redondance.

Notre analyse prédit que les locuteurs contemporains dérivent les X-t-eur des X-t-ion. Pour savoir si les prédictions de cette analyse sont justes, il faut examiner les néologismes et voir dans quelle mesure ils les confirment. L'examen des néologismes représente à notre avis le test le plus déterminant pour une analyse de ce genre qui, se situant sur un plan synchronique, ne vise pas à décrire l'histoire de la morphologie du français mais à rendre compte des règles que les locuteurs actuels utilisent.

\subsubsection{Le test de la néologie}

Nous avons choisi deux corpus pour l'examen des néologismes: celui de Darmesteter $(1877,1975)$, qui s'étend sur un peu plus d'une centaine d'années (approximativement 1750-1875) et celui de Dubois (1962), qui prend la relève pour aller jusqu'à la moitié du $20^{\mathrm{e}}$ siècle. Darmesteter constitue le corpus le plus complet en ce qui concerne les années en question; quant à Dubois, il récapitule à peu près tout ce que nous trouvons chez d'autres auteurs pour l'époque contemporaine (chez Goosse 1975, par exemple, qui analyse les néologismes recensés par P. Gilbert (1971) dans son dictionnaire des mots nouveaux).

Nous allons donc examiner les néologismes et voir dans quelle mesure ils peuvent confirmer ou infirmer notre analyse. Notre analyse sera étayée si nous trouvons de nouvelles formes X-t-eur et si ces formes ont chacune un corrélat en -tion à partir duquel elles auraient pu dériver. Par contre, puisque nous supposons que ce sont les X-t-ion qui servent de bases aux X-t-eur, et non l'inverse, nous ne devrions pas trouver systématiquement une forme X-teur existante pour chaque nouvelle forme en -tion. D'autre part, nous comptons trouver peu de néologismes de type X-eur (ex.: assur-eur) ayant un corrélat $\mathrm{X}$-t-ion puisque nous supposons que la forme en -tion, lorsqu'elle existe, est la base privilégiée de la dérivation en -eur; néanmoins, le fait que la X-t-ion existe mais qu'elle n'ait pas servi de base au dérivé en -eur n'est en soi que la confirmation qu'il y a deux bases pour la dérivation en -eur: Le radical verbal ou le nom en tion.

\subsubsection{Nouvelles formes $X$-t-eur}

Chez Darmesteter (1877) :

(25) absoluteur, accélérateur, acclamateur, adjudicateur, aérateur, animateur, annonciateur, aspirateur, assimilateur, aviateur, civilisateur, clavilisateur, coagulateur, colonisateur, commu- 
tateur, concentrateur, condensateur, congélateur, coordinateur, copulateur, déformateur, déviateur, dislocateur, élucubrateur, émasculateur, épurateur, évaluateur, évangélisateur, extirpateur, filateur, insufflateur, injecteur, jugulateur, mensurateur, nomificateur, monopolisateur, panificateur, percolateur, réacteur, régulateur, retardateur, réorganisateur, scarificateur, triturateur, volateur, vulgarisateur.

Chez Dubois (1962) (nous n'avons pas repris les néologismes qui se trouvaient déjà chez Darmesteter) :

(26) activateur, adapteur, autotransformateur, combinateur, évacuateur, excavateur, excommunicateur, hachurateur, inséminateur, ozonateur, répurgateur, sanguiformateur, saturateur, sustentateur, variateur.

Sur les 61 formes $\mathrm{X}$-t-eur ci-dessus, cinquante-six ont un corrélat X-t-ion attesté. Les cinq exceptions sont : clavilisateur, combinateur, filateur, hachurateur et volateur. Disons tout de suite à propos de clavilisateur et de volateur que ces formes n'apparaissent dans aucun des ouvrages de référence que nous avons consultés. Peut-être avaient-ils des corrélats en -tion au moment de leur création, nous ne le savons pas. Hachurateur n'a pas de corrélat en -tion dans nos ouvrages de référence, bien que certaines personnes semblent convaincues que l'opération consistant à effectuer des hachures sur une figure à l'aide d'un hachurateur est l'hachuration, ce qui prouve que hachuration est un mot possible. La seule explication valable à notre avis, pour la formation de filateur et de combinateur, est ce que Goosse appelle la substitution de suffixes, type de dérivation très productive en français. Ainsi, filateur dériverait de filature (-ure/-eur), et combinateur de combinatoire (-oire/-eur). On peut supposer qu'on ait cherché le mot en -tion mais que, faute de cette base, on se soit rabattu sur le radical savant qui, d'habitude, est celui qui se retrouve dans les mots en -tion mais qui sert également à former les mots en -ture, -toire, etc. Nous pensons que cinquante-six cas clairs sur soixante et un constituent une proportion assez significative en faveur de notre hypothèse.

\subsubsection{Nouvelles formes $X$-t-ion}

Chez Darmesteter (1877) :

(27) absorbation, accrémentition, acétification, actualisation, aération, annexation, arrosion, aviation, célébration, centralisation, claustration, colorisation, compromission, conglomération, constatation, déblatération, dégénération, dégoûtation, démoralisation, domestication, formication, frelatation, germanisation, hellénisation, idéalisation, individualisation, irisation, localisation, majoration, momification, numérotation, ornementation, prussification, réglementation, réorganisation, revaccination, romanisation, solidarisation, spication, unification, verrition, vulgarisation.

Chez Dubois (1962) : Il s'impose ici de dire que Dubois nous donne environ cent cinquante nouvelles formes en -tion. Mais, à l'inverse de Darmesteter qui distingue les vraies créations françaises des emprunts, Dubois n'opère aucun classement. Il y a donc une distinction à faire chez Dubois entre les vraies dérivations en -tion et les autres qui constituent soit des emprunts (à l'anglais notamment, ex.: automation), soit des formes dérivées par adjonction d'un préfixe à un mot en -tion déjà existant (ex.: microdissection), soit des formes obtenues par ce qui semble être une substitution de préfixes (ex.: déflation, d'inflation). Bien qu'il soit difficile quelquefois de dire avec précision si telle forme est un emprunt à l'anglais où on la trouve également, il semble que l'on 
puisse avancer qu'au moins un tiers de ces formes constituent des emprunts directs à cette langue. Les formes dérivées par adjonction d'un préfixe au mot en -tion et celles obtenues par ce que nous appellerons provisoirement une substitution de préfixes constituent un autre tiers. Une cinquantaine de formes, soit le tiers restant, sont les seules qui peuvent être retenues comme dérivations originales françaises en -tion. Ces dérivés, formés par suffixation cle -ation, se répartissent eux-mêmes en deux types: un peu moins d'une quarantaine qui doivent leur existence à un verbe Xiser (ex. : scolariser/scolarisation, vulgariser/vulgarisation), que nous ne reprendrons pas en détail étant donné la transparence de leur formation, et les autres, au nombre de treize, qui sont les suivants :

(28) carbonatation, cryoturbation, décaféination, décuscutation, démargarination, évagination, exonération, ignifugation, nitration, nitratation, nitrosation, polygonation, pyrogénation.

En ce qui concerne les treize formes ci-dessus, il semble qu'elles ne peuvent dériver de formes en -teur, puisqu'elles n'ont pas de corrélat X-t-eur. D'autre part, en ce qui concerne les formes X-t-ion correspondant à un verbe Xiser, type de formes que nous avons trouvées chez Dubois, elles sont très productives à partir de ce suffixe verbal, car théoriquement on peut avoir un mot en -ation à partir de tout verbe en -iser où -iser a une fonction sémantique factitive, ce qui écarte la possibilité qu'elles dérivent de formes $\mathrm{X}$-t-eur. Voici, à titre d'exemples, quelques-unes de ces formes à partir desquelles on peut facilement retrouver la base verbale Xiser :

(29) commercialisation, décolonisation, hygiénisation, homogénéisation, numéralisation, rationalisation, standardisation, etc.

Si l'on examine les quarante-deux X-t-ion de Darmesteter en (27), on s'aperçoit que, dans la majorité des cas, la X-t-eur n'existe pas (mais, bien entendu, pourrait exister), ce qui confirme que les $\mathrm{X}$-t-eur dérivent des $\mathrm{X}$-tion et non l'inverse.

\subsubsection{Nouvelles formes $X_{v}$-eur}

Chez Darmesteter (1877) et Dubois (1962) : comme nous l'avons déjà souligné, ce type de dérivation est très productif en français. Darmesteter à lui seul nous donne plus d'une soixantaine de cas et Dubois en recense une cinquantaine. Nous épargnerons au lecteur les listes de ces mots qu'on pourra reconstituer en se reportant aux auteurs.

Voici seulement quelques exemples :

(30) acclimateur, amincisseur, aplatisseur, approfondisseur, assainisseur, atermoyeur, bagarreur, baptiseur, bougeur, bénisseur, biseauteur, blagueur, boxeur, caramboleur, circuleur, chapardeur, chippeur, collectionneur, etc.

En parcourant ces formes $\mathrm{X}_{\mathrm{v}}$-eur qui dépassent la centaine, nous trouvons sept mots qui peuvent être corrélés à des formes en -tion; ce sont :

(31) activeur (activation), acclimateur (acclimatation), circuleur (circulation), collectionneur (collection), condenseur (condensation), confectionneur (confection), démargarineur (démargarination).

Il s'impose de noter qu'activateur, collecteur et condensateur existent aux côtés d'activeur, de collectionneur et de condenseur. Ces mots prouvent, comme nous l'avons dit, l'existence des deux bases et l'une ou l'autre peut servir à la dérivation des mots en -eur. Notre analyse prédit que la base privi- 
légiée est $\mathrm{X}$-t-ion, si elle existe, et cela se confirme par le nombre de dérivés qui suivent cette tendance; car finalement si on fait abstraction d'activeur, de collectionneur et de condenseur, nous n'avons que quatre exceptions sur plus d'une centaine d'exemples, exceptions pour lesquelles le mot en -teur est souvent possible, bien que non existant, et qui peuvent s'expliquer par la grande productivité des formes $\mathrm{X}_{\mathrm{v}}$-eur.

\subsubsection{Néologismes récents}

Nous aimerions maintenant faire état de trois créations néologiques récentes qui sont la confirmation de la productivité de la dérivation des X-t-eur à partir des X-t-ion.

Nous avons été le témoin de la création par le Gouvernement du Québec du néologisme locateur qui a dû être formé à partir de location. Ce terme apparaît sur le bail standard de la Régie des loyers à partir de 1977. D'autre part, à titre de traducteur-terminologue d'une entreprise de presse, nous avons assisté personnellement à la création du néologisme manutenteur à partir de manutention. Le syndicat à majorité francophone des «Paperhandlers I.P.G.C. Union of Montreal, local 41 », a choisi ce terme pour désigner ses membres envers et contre tous les traducteurs qui proposaient à la place «préposé à la manutention du papier» ou «manutentionnaire de papier». Finalement, nous trouvons dans un document intitulé Consultations sur la politique des stages de l'UQAM, février 1981, les lignes ci-dessous :

Ces politiques pensées pour chaque programme dans le sens d'un document d'information à l'intention des «acteurs» du stage (étudiants, professeur responsable, agent de stages, professionnel associé), viendraient assurer un caractère de continuité que ne comporte pas actuellement le syllabus de cours. (p. 8)

Dans ces lignes, le mot acteurs, entre guillemets, a retenu notre attention. Ce qui est intéressant de constater, c'est que ce mot a été dérivé, selon toute vraisemblance, de action en dépit du fait que le mot acteur était déjà lexicalisé avec un sens bien particulier: "celui qui joue un rôle". Cela prouve à notre sens la productivité de ce processus qui a eu raison d'un obstacle assez grand, celui de la forme en -teur lexicalisée.

Il semble donc raisonnable de conclure que les néologismes que nous avons vus concourrent à la vérification de notre hypothèse et confirment la productivité des X-t-eur à partir des X-t-ion : en effet, sur soixante et un néologismes $\mathrm{X}$-teur, cinquante-six dérivaient clairement d'une base X-t-ion, sur une centaine de néologismes X-tion, très peu pouvaient être corrélés à une X-t-eur, ce qui détermine le sens de la règle (X-t-eur dérive de $\mathrm{X}$-t-ion et non l'inverse); sur plus d'une centaine de nouveaux mots en -eur, seuls quatre avaient comme corrélat un mot X-tion, ce qui montre que la base X-t-ion, si elle existe, est la base privilégiée pour la dérivation en -eur. Le test de la néologie s'est par conséquent révélé très positif.

\subsection{Les exceptions dans l'inventaire lexical}

Nous venons de voir la néologie qui, à notre avis, est le test le plus déterminant pour notre hypothèse. Nous voulons maintenant nous rendre compte des 
données sur lesquelles repose l'analyse que font les locuteurs contemporains quand ils considèrent que les X-t-eur dérivent des X-t-ion. Il nous faut donc voir s'il existe pour chaque mot en -teur de notre vaste corpus, constitué principalement par le dictionnaire de Juilland (1965) et par diverses autres sources, un mot X-t-ion.

Nous avons effectivement des exceptions. Ces exceptions se répartissent en deux groupes : les X-t-eur sans base $\mathrm{X}$-t-ion et les $\mathrm{X}_{\mathrm{v}}$-eur avec corrélat X-t-ion.

\subsubsection{Les X-t-eur sans base X-t-ion}

Voici ci-dessous les mots qui constituent, à notre connaissance, toutes les exceptions de ce type :

(32) accompagnateur amateur

armateur

blasphémateur

calculateur

calomniateur

commentateur
cultivateur
dessinateur
dispensateur
lecteur
ordonnateur

sauveteur

scrutateur

serviteur

triomphateur

Xculteur (agriculteur, pisciculteur, etc.)

Certains de ces mots constituent des emprunts directs au latin, selon le Petit Robert (1973) (cela dit à titre d'explication, car nous admettons que synchroniquement ils constituent des exceptions) :

(33) amateur, de amator, $15^{\mathrm{e}}$ siècle

armateur, de armator, $16^{\mathrm{e}}$ siècle

calculateur, de calculator, $16^{\mathrm{e}}$ siècle

lecteur, de lector, $12^{\mathrm{e}}$ siècle

scrutateur, de scrutator, $14^{\mathrm{e}}$ siècle

calomniateur, de calomniator, $13^{\mathrm{e}}$ siècle

dispensateur, de dispensator, $12^{\mathrm{e}}$ siècle

triomphateur, de triomphator, $14^{\mathrm{e}}$ siècle

En ce qui concerne le paradigme Xculteur, il faut noter qu'agriculteur est un emprunt direct au latin (mais qui pourrait synchroniquement être décrit comme dérivé d'agriculture par substitution de suffixes):

(34) agriculteur, de agricultor, $15^{\mathrm{e}}$ siècle

Nous pouvons admettre que les autres mots du genre apiculteur, ostréiculteur, pisciculteur, et sylviculteur ont été formés par analogie d'après le modèle agriculteur.

Nous enregistrons un emprunt à l'italien (Petit Robert, 1973) :

(35) dessinateur, de designatore, $17^{\mathrm{e}}$ siècle

Restent donc les mots suivants, qui ne peuvent pas être considérés comme emprunts (Petit Robert, 1973) :

(36) accompagnateur, d'accompagner, $17^{\mathrm{e}}$ siècle

blasphémateur, de blasphème, $14^{\mathrm{e}}$ siècle

commentateur, de commenter, $14^{e}$ siècle

cultivateur, de cultiver, $14^{e}$ siècle

ordonnateur, d'ordonner, $16^{\mathrm{e}}$ siècle

sauveteur, de sauvetage (subs. de suff.), $19 \mathrm{e}$ siècle

Nous avons donc dix-huit contre-exemples (ou un peu plus d'une vingtaine si l'on tient compte de toutes les formes de type Xculteur qui sont des dérivations savantes), pour la plupart des emprunts lexicalisés depuis longtemps. Nous pouvons donc dire que les locuteurs du français contemporain font l'analyse «X-t-eur dérive de $\mathrm{X}$ - $\mathrm{t}$-ion» sur la foi de centaines de paires correspondantes, malgré ce nombre infime d'exceptions. 


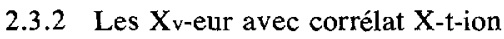

Nous avons trouvé huit mots en -eur qui ne sont pas dérivés de la $\mathrm{X}$-t-ion correspondante, comme on pourrait s'y attendre, mais directement $\mathrm{du}$ radical verbal. Voici ces mots ci-dessous :

$\begin{array}{clll}\text { (37) a) décanteur } & \text { (décanter) } & \text { ?décantateur } & \text { (décantation) } \\ \text { exploiteur } & \text { (exploiter) } & \text { *exploitateur } & \text { (exploitation) } \\ \text { orienteur } & \text { (orienter) } & \text { ?orientateur } & \text { (orientation) } \\ \text { planteur } & \text { (planter) } & \text { *plantateur } & \text { (plantation) } \\ \text { b) acquéreur } & \text { (acquérir) } & \text { *acquisiteur } & \text { (acquisition) } \\ \text { nageur } & \text { (nager) } & \text { *natateur } & \text { (natation) } \\ \text { nourrisseur } & \text { (nourrir) } & \text { ?nutriteur } & \text { (nutrition) } \\ \text { pollueur } & \text { (polluer) } & \text { *polluteur } & \text { (pollution) }\end{array}$

Avant d'examiner en détail ces exceptions, nous tenons à souligner que certains locuteurs trouvent parfois possibles, bien qu'inexistants, les mots en -teur que nous signalons ci-dessus comme douteux ou agrammaticaux. Ainsi, certains locuteurs admettent par exemple orientateur au même titre qu'orienteur. De plus, nous devons rapporter que nous avons entendu à la radio le mot nutriteur, utilisé pour parler d'un engrais: ce mot qui n'est nulle part attesté constitue une innovation, peut-être même spontanée, signe de la productivité de la dérivation en -teur à partir de X-t-ion, car nous sommes d'avis que c'est le mot nutrition qui a dû lui servir de base.

Nous allons maintenant examiner nos exceptions. Nous avons placé dans le groupe a) les mots en -eur dont le corrélat X-t-ion finit en -tation, le premier / $t$ / étant la finale du radical verbal. On pourrait se demander a priori si nous n'avons pas affaire à une contrainte phonotactique qui empêche la formation de mots en -tateur, ou du moins une tendance en français à réduire cette séquence à -teur. Voici ci-dessous des exemples de mots en -tateur illustrant (à notre connaissance) tous les contextes phonologiques d'apparition de cette séquence qui montrent qu'il ne semble pas y avoir de difficulté à dériver une X-tateur d'une X-tation.

$\begin{array}{llll}\text { (38) } \begin{array}{l}\text { agitateur } \\ \text { annotateur }\end{array} & \text { exportateur } & \text { commutateur } & \text { manifestateur } \\ \text { argumentateur } & \text { fomentateur } & \text { dégustateur } & \text { récitateur } \\ \text { captateur } & \text { importateur } & \text { excitateur } & \text { sanitateur } \\ \text { citateur } & \text { incitateur } & \text { expérimentateur } & \text { tentateur }\end{array}$

On pourrait peut-être se demander, avant d'examiner les contextes phonologiques, si la réduction de -tateur à -teur n'est pas fonction de la longueur du mot, c'est-à-dire de son nombre de syllabes. Il semble qu'il faille écarter cette possibilité car, nous pouvons le voir par les exemples ci-dessus, le nombre de syllables ne parait jouer aucun rôle: il y a des mots dont -tateur est précédé d'une, de deux, de trois ou même de quatre syllables. Serait-ce alors le contexte phonologique précédant -tateur qui détermine la troncation du segment -at-(orientateur)? Nous présentons ci-dessous les contextes d'apparition de nos exceptions suivis de ceux des formes qui terminent par le segment-tateur.

Contexte d'apparition de nos exceptions :
(39)

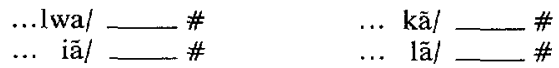

2. Mais nous avons inquisiteur/inquisition (où il n'y a aucun corrélat verbal). 


\section{Contexte d'apparition des formes en -tateur existantes :}

$(40)$

$$
\text { ...mand }
$$

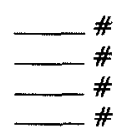

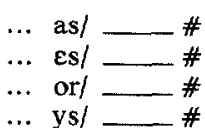

Nous remarquons que /ã/ précède aussi bien -teur que -tateur. Seul /a/ semble exclusif à -teur. Toutefois, le phonème qui précède /ã/ pour nos exceptions ne se retrouve pas dans les formations en -tateur; en vertu de cela, et en faisant abstraction de notre intuition linguistique, nous pourrions déclarer que la séquence -at- subit une troncation lorsque le $/ t /$, qui appartient au radical, est précédé soit de /a/, soit de / $\underset{1}{\mathbf{k}}$ ã/. La règle que nous formulerions nous permettrait donc de «régler» ces quelques cas en les faisant dériver du mot en -tion correspondant. Pourtant, nous renonçons à cette hypothèse qui repose sur une distribution phonologique que nous considérons purement fortuite. Jusqu'à preuve du contraire, nous admettrons que les quatre formes en (37)a) dérivent du verbe malgré le mot en -tion existant. Ainsi, nous supposons que orienteur ne dérive pas d'orientation, mais d'orienter. Nous tiendrons le même raisonnement pour les formes en (37)b) et dirons qu'elles dérivent du verbe en dépit de l'existence du mot en -tion. Comme nous l'avons déjà dit précédemment, notre analyse n'exclut pas que des agentifs en -eur dérivent du verbe; au contraire, c'est sur cette coexistence de bases que repose notre analyse et qui explique la présence de paires du genre receveur $\sim$ récepteur, transformeur transformateur, etc. Notre hypothèse prédit cependant que lorsque la forme en -tion existe, c'est elle qui fournit la base privilégiée de la dérivation en -eur. Il semble qu'en se fondant sur l'existence des paires synonymes du genre receveur récepteur, on puisse affirmer que certaines paires de ce genre ont coexisté un certain temps jusqu'à ce que l'un des mots ait été lexicalisé.

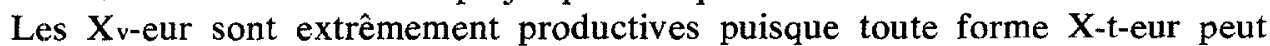

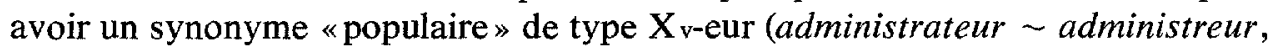
réparateur répareur) alors que l'inverse n'est pas vrai, la condition sine qua non de l'existence de la X-t-eur étant l'existence de la X-t-ion; c'est cette productivité presque absolue de $X_{v}$-eur qui pourrait faire qu'une fois qu'une $X_{v}$-eur est lexicalisée, elle bloque généralement la dérivation de la $X$-t-eur à partir de son corrélat en -tion.

\subsubsection{Une contrainte pour la dérivation de $\mathrm{X}$-t-eur à partir de $\mathrm{X}$-t-ion?}

Il existe, semble-t-il, une contrainte qui interdit la formation de X-t-eur à partir de $\mathrm{X}$-t-ion où $\mathrm{X}$-t-ion est dérivée d'un verbe de type finir. Considérons les exemples ci-dessous :

(41) *aboliteur (d'abolition) _ mais abolisseur (d'abolir)

*démoliteur (de démolition) - mais démolisseur (de démolir)

* finiteur (de finition) - mais finisseur (de finir)

* puniteur (de punition) _ $\quad$ mais punisseur (de punir)

$\grave{A}$ priori, cette contrainte pourrait constituer une violation de la condition d'adjacence de Siegel (1974) qui énonce qu'une règle de formation de mots ne peut pas lire au-delà du cycle adjacent à celui où a lieu la suffixation. Étant 
donné que la suffixation en -tion pourrait être considérée comme appartenant à un cycle précédent, la règle d'adjonction de -eur ne devrait pas être intéressée par le type de radical ayant servi de base.

Cependant, nous avons montré dans Di-Lillo (1981: 95 et suiv.) que les noms d'action qui correspondent aux formes de type finisseur ne sont pas des formes en -tion malgré leur apparence (ex. : finition). En fait ces formes, qui pouvaient être des formes en -tion à un stade d'évolution du français, sont aujourd'hui analysées par les locuteurs contemporains comme formes dérivées du radical long du verbe, ex. : finiss +ion; cette confusion peut être mise sur le compte de l'orthographe. L'analyse qui fait d'un mot comme finition le dérivé du radical long du verbe relève de la généralisation du principe qui veut qu'en français ce soit le radical long qui serve de base à la dérivation.

Toutefois, il nous faut faire état d'un contre-exemple à cette "contrainte». En effet, dans le texte ci-dessous, nous trouvons ( $2^{\mathrm{e}}$ ligne) définiteur, dérivé sans nul doute de définition, malgré le radical définiss- :

Épigones des chamanes d'autrefois, les fonctionnaires exercent une double fonction de communication: d'abord en tant que définiteurs (souligné par nous) de situation, maitres du discours, interprètes du sens inscrit dans le monde nouveau; en outre, ils tissent un pont entre les Esquimaux et les Puissances invisibles: l'État, les Blancs. (J.J. SIMARD, «Terre et pouvoir au Nouveau-Québec», in Études/Inuit/Studies, vol. 3, n 1, 1979, Université Laval, p. 111).

Le contre-exemple ci-dessus est un indice incontestable de la productivité de la dérivation des $\mathrm{X}$-t-eur à partir des $\mathrm{X}$-t-ion.

\section{CONCLUSIONS DE LA DEUXIËE PARTIE}

Nous venons de montrer, dans cette deuxième partie, la complexité à laquelle on doit faire face lorsqu'on veut décrire les X-t-eur d'une façon traditionnelle, c'est-à-dire en les faisant dériver d'un radical verbal, d'autant plus que quelquefois ce radical verbal n'existe même pas. Nous avons proposé à la place de les faire dériver de la X-t-ion qui généralement existe à côté de cette $\mathrm{X}$-t-eur. Nous avons fait état des exceptions qu'on retrouve tant au plan de la néologie qu'à celui des formes lexicalisées. Ces exceptions, en fait, ne sont pas assez nombreuses pour infirmer le traitement que nous proposons; de plus ce nombre infime de contre-exemples ne font que montrer que, malgré leur existence, les locuteurs ont fait synchroniquement l'analyse «X-t-eur dérive de $\mathrm{X}$-t-ion», en se fondant sur la masse énorme de paires régulières. Dans la dernière et troisième partie de cet article nous nous pencherons sur le côté théorique du traitement que nous préconisons et tenterons de proposer une formalisation adéquate des règles que notre hypothèse met en jeu; notamment nous verrons comment nous pouvons intégrer notre analyse dans un modèle récent de la morphologie, celui de Lieber (1980).

Antoine Di-Lillo

RÉFÉRENCES : voir Meta, vol. 27, n 3, p. 330. 